\title{
Diffusion des images radar et satellite
}

\section{Broadcasting radar and satellite pictures}

\author{
C. Gaillard \\ Direction de la Météorologie \\ Etablissement d'Etudes et de Recherches Météorologiques \\ Centre de Recherche en Physique de l'Atmosphère \\ 78470 Magny-les-Hameaux
}

\section{Introduction}

Les premiers radars météorologiques sont apparus dans les années 50. L'information donnée par ces appareils est encore souvent distribuée suivant un procédé inchangé. Un opérateur décrit l'image radar et le texte ainsi obtenu est diffusé sous forme de messages. Ce système présente de nombreux inconvénients. Le positionnement des échos ainsi que leurs contours sont difficiles à décrire, et à ces imprécisions s'ajoutent les erreurs humaines toujours possibles; en outre, le temps nécessaire à la diffusion de l'information est trop important (durée supérieure à la demi-heure) en regard de la nature éminemment changeante des informations radar.

En 1970, à Danmartin en Goëlle un radar de type MELODI est installé et équipé d'une transmission fac similé vers les stations météorologiques de Roissy, Paris, Le Bourget et Orly. La carte radar est dessinée à la main puis transmise par une ligne téléphonique. Les délais sont considérablement améliorés (moins de 10 minutes) mais une imprécision persiste dans le tracé. D'autre part, ce procédé ne permet pas de traitement informatique, d'où l'impossibilité d'envisager une centralisation efficace des images radar.

Les progrès de l'électronique et de la micro-informatique avec l'arrivée des microprocesseurs vont permettre de numériser l'image radar à un prix abordable. En même temps les améliorations et la banalisation des lignes de transmission téléphoniques vont faciliter l'acheminement des informations codées.

En 1980, une première chaîne de numérisation de signaux radar est installée à Dammartin en Goëlle. La chaîne SAPHYR fabriquée autour d'un système à microprocesseur 8080 monté sur des cartes au standard IEEE 796 n'est alors utilisée que pour l'acquisition de données sur bandes magnétiques.

En 1981, une console de réception, conçue à partir du même appareil, est mise en place au Centre Météoro- logique de Roissy; elle permet de visualiser l'image sans aucun traitement.

En 1982, l'adjonction d'un clavier permet d'accéder à différentes fonctions de traitement de l'image sur cette station de réception (pointeurs, fond de carte, isolignes, calculs de vitesse de déplacement, prévision). Simultanément, le réseau a été étendu aux stations météorologiques du Bourget, de Paris, d'Orly, puis début 1983 à la DDE de la Seine St-Denis. En mai 1983, une dernière station sera installée à Bordeaux. Le système SAPHYR est limité aux images radar. Parallèlement, une équipe de l'EERM Lannion a mené à bien la conception et la réalisation d'une chaîne de réception de l'image satellite transmise sur ligne téléphonique depuis le Centre de Lannion. Il s'agit de la chaîne ELVIR.

La société THOMSON commercialise par ailleurs une console de réception des images émises par les radars RODIN, le MR 750.

Il n'était pas souhaitable tant au point de vue financier qu'au point de vue technique, de doter les stations météorologiques d'appareils différents, ayant des fonctions presque identiques et alimentés par des lignes téléphoniques différentes.

Le développement de la diffusion de l'imagerie passait donc par la centralisation des différentes données pour permettre une insertion dans le réseau existant de transmission fac-codé, et par la conception d'un équipement unique que la Météorologie Nationale a décidé de faire industrialiser sous le nom de METEOTEL. Ce système devra permettre la réception des images radar MELODI et RODIN ainsi que l'imagerie satellitaire.

Ce matériel a fait l'objet d'un marché dont le cahier des charges a été défini à partir de l'expérience acquise lors de la réalisation de la chaîne SAPHYR actuellement en activité, et dont nous allons présenter les caractéristiques techniques et les différentes possibilités d'utilisation.

LA HOUILLE BLANCHE/ $\mathrm{N}^{\circ}$ 5/6-1983 


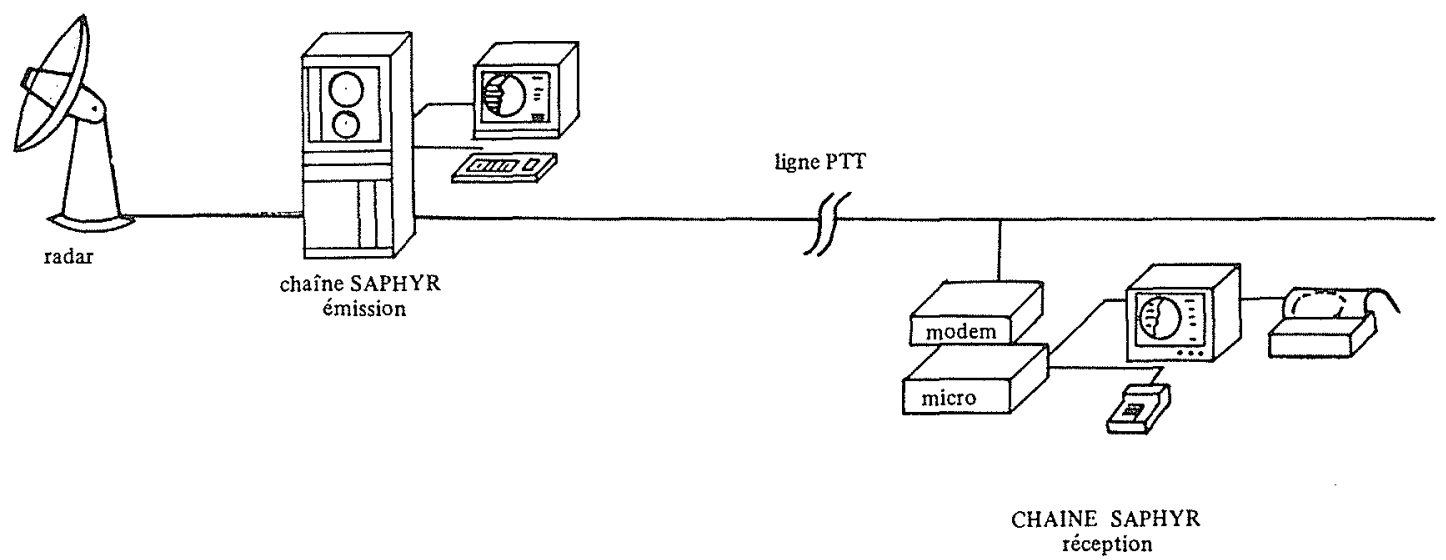

Figure 1 - Schéma de principe du système SAPHYR

\section{La chaine SAPHYR}

Le système SAPHYR est composé d'une chaine d'émission et d'une chaîne de réception reliées entre elles par une ligne PTT (Fig. 1).

\section{II.1. Description du matériel}

La chaîne d'émission assure les fonctions suivantes :

- commande de l'antenne,

- digitalisation du signal vidéo émis par le radar MELODI,

- enregistrement éventuel des données sur bandes magnétiques pour un traitement ultérieur,

- fabrication d'une image digitale des échos

L'image obtenue est transmise de manière automatique, toutes les quinze minutes par une ligne PTT. Les chaînes de réception raccordées à cette ligne ont pour buts :

- la restitution de l'image digitale sur un moniteur couleur

- l'exploitation de l'information

Le matériel, tant d'émission que de réception, a été conçu à partir des mêmes cartes standard montées sur le BUS IEEE 796.

La chaîne de réception est constituée de quatre éléments :

- un MODEM $4800 \mathrm{~b} / \mathrm{s}$ relié à une ligne spécialisée

- un bac calculateur contenant :

- une carte processeur dotée d'un microprocesseur 8080 , de 4 Koctet de RAM et de 16 Koctet de ROM.

- une carte vidé RAM ayant une résolution de $256 \times$ 256 points codés sur 4 bits adressable en $X$ et $Y$ et attaquant un moniteur couleur en RVB par l'intermédiaire d'une prise PERITEL.

- un moniteur couleur

- un clavier 16 touches

- en option, une imprimante graphique

\section{II.2. Visualisation de l'image}

La chaîne ainsi montée reçoit l'image envoyée par ligne PTT, image constituée de 256 lignes de 256 points codés sur 16 niveaux à la vitesse de $4800 \mathrm{~b} / \mathrm{s}$ en mode synchrone 8 bits. Cette image est transmise, ligne par ligne, en partant du haut de l'image avec synchronisation entre chaque ligne. La transmission débute aux minutes $0,15,30$ et 45 et dure environ 1 minute.

Le processeur scrute en permanence la ligne PTT pour détecter l'octet marquant le début de transmission d'une ligne. Il teste alors le code image, lit le numéro de la ligne à visualiser ainsi que le nombre d'octets à suivre, puis décode les 128 octets contenant les niveaux de 256 points de la ligne en les affectant sur l'écran. L'image ainsi obtenue est recopiée sur l'imprimante si celle-ci est connectée, et se présente suivant le schéma de la figure 2.

Chaque point de l'image couvre une superficie de $2,6 \times 2 \mathrm{~km}^{2}$. Le paramètre représenté est le facteur de réflectivité radar $Z$, exprimé en décibels $(d B Z)$ par rapport à l'unité couramment employée en météorologie, le $\mathrm{mm}^{6} \mathrm{~m}^{-3}\left(d B Z=10 \log _{10} Z, Z\right.$ en $\left.\mathrm{mm}^{6} \mathrm{~m}^{-3}\right)$. Les niveaux de réflectivité, au nombre de 16 , ne sont visualisés que sur 8 couleurs allant du gris au rouge, ceci dans un souci de clarification de l'image. Le Tableau I donne l'échelle des niveaux utilisés.

Tableau I-correspondances entre les couleurs, le facteur de réflectivité et l'intensité de la pluie applicables à la chafne SAPHYR. Les intensités de pluie $(R)$, obtenues par la relation standard $Z=200 R 1,6$, doivent être interprétées avec précautions

\begin{tabular}{|l|c|c|}
\hline Couleurs & $\begin{array}{c}\text { Facteur de } \\
\text { réflectivité } \\
\text { (en dbs) }\end{array}$ & Pluie en $(\mathrm{mm} / \mathrm{h})$ \\
\hline gris foncé & seuil à 16 & 15 \\
cyane & 17 à 23 & .4 \\
bleu clair & 24 à 32 & 1 \\
bleu foncé & 33 à 39 & 4 \\
vert & 40 à 46 & 11 \\
jaune & 47 à 54 & 25 \\
marron & 55 à 62 & 100 \\
rouge & 63 et plus & 300 \\
\hline
\end{tabular}

Chaque point représente la réflectivité à une altitude variable en fonction de la distance de ce point au radar, en raison de la rotondité de la terre, et du site du faisceau radar (Tableau II). 


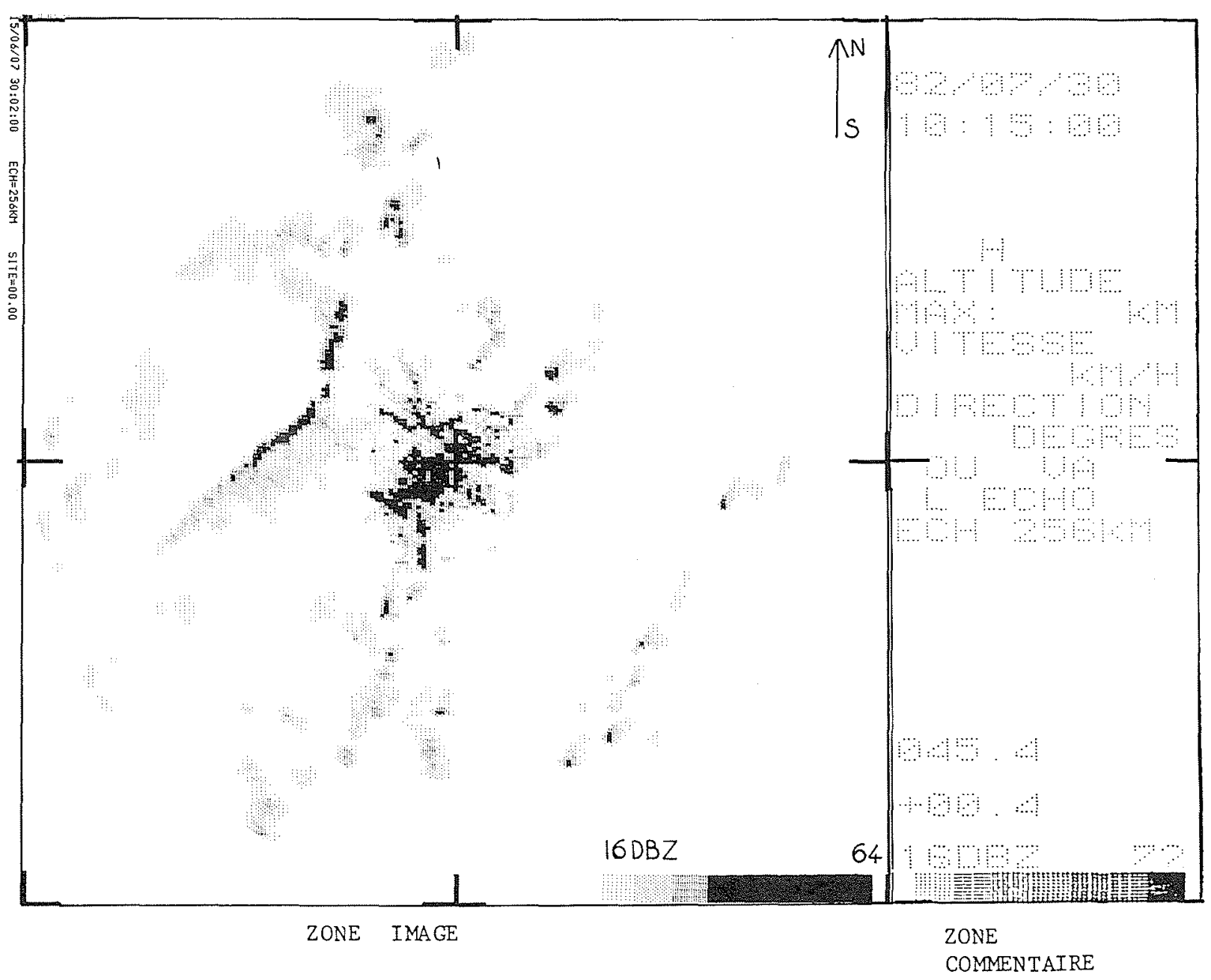

Figure 2

Tableau II - Altitude du faisceau radar en fonction de la distance pour un site de $0,4^{\circ}$

\begin{tabular}{|c|c|c|c|c|c|}
\hline$r$ en $\mathrm{km}$ & 50 & 100 & 150 & 200 & 250 \\
$h$ en $\mathrm{km}$ & 0.2 & 0.75 & 1.7 & 3.0 & 4.8 \\
\hline
\end{tabular}

La correction des niveaux des échos en fonction de la distance est faite automatiquement jusqu'à $100 \mathrm{~km}$ par le radar MELODI. Au delà, la correction à apporter est de $6 \mathrm{dbz}$ pour une distance de $200 \mathrm{~km}$ et de $8 \mathrm{dbz}$ pour une distance de $256 \mathrm{~km}$. Cette correction n'a pas été réalisée car elle n'apporte pas de précision supplémentaire pour l'exploitation météorologique de l'information.

Les images SAPHYR comportent sur la droite de l'écran une zone de "commentaires" de 256 lignes de 64 points contenant la date, l'heure et le site de l'image affichée, les commentaires transmis par l'opérateur radar, la mire et les résultats des différentes commandes.

\section{II.3. Exploitation de l'image}

En dehors des périodes de transmission, l'opérateur dispose de plusieurs commandes de traitement de l'image accessibles à partir des touches du clavier :

- positionnement géographique : cette commande fait apparaître un fond de carte en superposition de l'image radar. Ce fond de carte peut être différent suivant les besoins des utilisateurs.

- positionnement du pointeur : cette commande gère un pointeur se déplaçant en coordonnées cartésiennes ou en coordonnées polaires dans un repère ayant pour origine soit l'emplacement du radar, soit un point choisi par l'utilisateur. On obtient simultanément un agrandissement par 2 de la zone entourant le pointeur.

- mémorisation des échos : cette commande mémorise les contours des échos.

La taille mémoire de 4 Koctets du système ne permet pas de mémoriser une image entière nécessitant $32 \mathrm{Koc}$ tets. Il est apparu aussi intéressant de ne stocker que les contours des échos, opération qui utilise moins de mémoire.

- tracé des échos mémorisés : cette commande trace en superposition de l'image radar les contours des échos mémorisés par la commande précédente.

- déplacement des échos mémorisés : cette commande permet le déplacement des contours des échos mémorisés afin de les amener en coincidence avec les échos de la dernière image radar. La vitesse et la direction du déplacement sont indiquées dans la zone commentaire. Cette opération peut être répétée autant de fois que l'image comporte d'échos différents.

- extrapolation du déplacement des échos : cette com- 
mande permet d'extrapoler par pas de 5 min. la position des échos à partir de la vitesse d'advection calculée par la commande précédente ou fournie par l'opérateur radar.

La précision du calcul de la vitesse d'advection des échos est limitée par la résolution spatiale de l'image $(2 \times 2,6 \mathrm{~km})$. La déformation temporelle des échos aggrave cette imprécision puisqu'elle empêche de comparer des images trop espacées dans le temps. Le prévisionniste devra choisir un écart de temps entre l'image et le contour stocké adapté à la situation météorologique.

Ces fonctions élémentaires n'exigent pas de supplément de matériel coûteux puisque 4 Kactets de mémoire RAM et un clavier 16 touches suffisent à les activer. Sans doute ne sont elles pas encore assez élaborées, mais elles constituent à nos yeux l'élément qui fait qu'une "belle" image devient un document de travail exploitable.

\section{METEOTEL}

La Météorologie souhaite doter ses stations départementales d'un équipement de visualisation industrialisé inspiré des chaînes de réception SAPHYR. Le matériel et le logiciel sont identiques à ceux de la chaîne SAPHYR à l'exception du microprocesseur 8 bit qui est remplacé par un matériel à 16 bit indispensable à l'accroissement de la taille mémoire. Par contre, METEOTEL pourra recevoir en option une plus grande variété d'images provenant de deux sources distinctes :

a) sur une ligne spécialisée elle recevra les images radars MELODI ou RODIN toutes les quinze minutes avec une résolution spatiale d'environ $2 \mathrm{~km} \times 2 \mathrm{~km}$

b) sur la ligne fac codé reliant l'ensemble des stations météorologiques au centre de Paris, transiteront les images suivantes en plus des cartes destinées au fac codé :

- image radar centralisée composée à partir de l'ensemble des radars français et de certains pays voisins toutes les 15 ou 30 minutes avec une résolution de $6 \times 6 \mathrm{~km}$,

- images radar centralisées prévues,

- image satellite visible ou par infrarouge au format identique ou images radar,

- image satellite visible ou par infrarouge couvrant l'Europe de l'Ouest avec une résolution de $12 \times 12 \mathrm{~km}$.

Toutes les images évoquées ci-dessus pourront également arriver via le réseau téléphonique commuté

Pour satisfaire l'ensemble des différentes stations météorologiques et des utilisateurs extérieurs éventuels, METEOTEL est composée d'une version de base qui pourra recevoir 6 options par simple ajoût de carte ou de périphériques sans aucune modification du câblage initial.

La version de base se compose :

- d'un chassis recevant un tableau de commande sur

la face avant,

- d'une carte processeur,

- d'une carte VIDEO RAM,

- d'un moniteur couleur.
Elle affichera en permanence sur l'écran la dernière image reçue, en couleur 8 niveaux pour les images radars et en noir et blanc 16 niveaux pour les images satellite. Un interrupteur placé sur la face avant permettra de bloquer la réception pour conserver une image particulièrement intéressante. Un deuxième interrupteur permettra d'afficher un fond de carte.

L'option 1 consiste en la fourniture d'un clavier 16 touches permettant d'accéder à toutes les fonctions de la chaîne SAPHYR actuelle. L'utilisation du panneau de commande de la face avant permettra de simplifier la manipulation des commandes.

L'option 2 consistera à augmenter la taille mémoire à 64 Ko rendant possible le stockage de l'image en mémoire.

L'option 3 concernera le branchement d'une imprimante.

L'option 4 se rapportera au branchement d'un magnétophone servant au stockage des images.

L'option 5 a pour but de faire passer la taille mémoire à 512 Koctets et à recevoir simultanément les images provenant de la ligne fac codé ou des radars locaux. L'augmentation de la capacité mémoire permettra de stocker 15 images. L'utilisateur pourra programmer à l'avance par l'intermédiaire d'un menu le nombre d'image par type qu'il désire recevoir et le type d'image qu'il désire visualiser.

L'option 6 est relative au remplacement d'une ligne spécialisée par une ligne téléphonique normale qui n'est utilisée que le temps d'une émission d'image.

\section{Conclusions}

Les chaînes METEOTEL dont nous venons de définir les caractéristiques devraient commencer à être installées dans les stations météorologiques à partir de juin 1984. Des utilisateurs extérieurs intéressés par la vitesse de diffusion de l'information météorologique pourront également se doter de ces stations.

Dans le domaine de la prévision à longue et moyenne échéance, le système METEOTEL ne sera d'aucune utilité sauf peut être dans l'avenir pour visualiser les résultats des modèles numériques. Par contre, il peut devenir un outil de travail puissant pour le prévisionniste des stations météorologiques départementales dont l'activité est principalement tournée vers la prévision locale à brève échéance. Toute prévision commence par une bonne analyse de la situation présente, ce qui implique un réseau d'observation adapté à l'échelle des phénomènes à prévoir.

Le réseau d'observation au sol et en altitude s'il permet de suivre la majorité des perturbations océaniques de grandes amplitudes peut, de par son inhomogénéité, sur les zones maritimes en particulier, laisser passer au travers de ses mailles des phénomènes de plus faibles envergures mais pourtant significatifs aux yeux de l'usager qui juge localement la qualité des prévisions. Nul doute que l'imagerie satellitaire diffusée presque instantanément sera d'une grande utilité pour situer avec précision la position d'un front, sa vitesse de déplacement ainsi que l'amorce d'ondulation souvent incommode à déceler sur les zones pauvres en stations d'obser. vations. 
Les phénomènes météorologiques comme les advections froides d'altitude génératrices de situations orageuses où les secteurs chauds occlus de faible étendue pourront être plus facilement repérés qu'autrefois. Mais c'est dans le domaine de la prévision à très brève échéance que l'apport des stations METEOTEL sera capital et permettra de satisfaire un nombre croissant d'utilisateurs soucieux d'optimiser leurs activités soumises aux intempéries. Dans ce cas, l'imagerie radar, renouvelée toutes les 15 minutes, deviendra le moyen privilégié permettant d'accéder aux phénomènes de petites échelles parfois très violents qui, jusqu'à présent, échappaient au domaine de la prévision.

\section{Abstract \\ Broadcasting radar and satellite pictures}

The extremely changing and transient nature of radar and satellite data required rapid broadcasting systems and frequent updating of information. New developments in telephone networks and microprocessing mean that such broadcasting can now be realised on a wide variety of equipment which can be low in cost but nevertheless capable of making adjustment and interpretation of pictures easy for users. The Meteorological office intends to have reception equipment for radar and satellite pictures marketed under the name of METEOTEL and supplied to a maximum number of stations in its network and previous users will also be able to install such equipment. This equipment has been designed on the basis of experience acquired in prototype stations constructed by the Meteorological Research Studies Establishment (l'Etablissement d'Etudes et de Recherches Météorologiques) some of which have been set up in the Paris region for experimental purposes. Different possible applications for these prototype stations are given together with the different functions which the METEOTEL stations will fulfil. 